\title{
Application of Spectral Element Method Combining Dilatation Theory to Sound Generated by a Co-rotating Vortex Pair
}

\author{
Zhenzhong Bao and Guoliang Qin \\ School of Energy and Power Engineering, Xi'an Jiaotong University, Xi'an, Shaanxi 710049, P. R. China.
}

(Received 17 October 2016; accepted 2 February 2018)

\begin{abstract}
A spectral element method for the simulation of an acoustic field is applied to the case of a co-rotating vortex pair in both stationary medium and mean flow. Based on the dilatation theory, the second time derivative of the pseudopressure is used as the source of the inhomogeneous convected wave equation which is discretized by a spectral element method in space and the Newmark- $\beta$ method in time marching. In addition, the nonreflecting boundary condition is adopted too. Then we compared the numerical results with the analytical solution. Numerical results are in good agreement with the analytical solution. Moreover, different grid spacings and time steps are investigated for evaluating the numerical accuracy. To study the frequency content of the sound, spectral analysis is also carried out. Finally, sound propagation in uniform flows and sheared mean flows are simulated and analyzed. This study shows the capabilities of the spectral element method combined with dilatation theory for the aeroacoustic problems.
\end{abstract}

\section{INTRODUCTION}

The acoustic analogy theory ${ }^{1}$ introduced by Lighthill to study jet noise is now over six decades, and it has been by far the most successful and versatile theory in dealing with aeroacoustic problems. Considering the pseudo-sound pressure, Ribner ${ }^{2,3}$ and Meecham ${ }^{4}$ offered a different approach to Lighthill's acoustic analogy which posited fluctuating fluid dilatations as the acoustic source. One motivation for developing a new expression is that the pseudo-sound term is much easier to calculate relative to Lighthill's stress tensor. According to this dilatation theory which also corresponds to the acoustic/viscous splitting technique derived by Hardin and Pope, ${ }^{5}$ Hurdle, ${ }^{6}$ Ribner and Crawley ${ }^{7}$ studied the aerodynamic noise generating from a jet engine. Combining large eddy simulation and dilatation theory, Flemming ${ }^{8}$ used a hybrid approach to study the combustion noise of a turbulent flame. Hiramoto et al. ${ }^{9}$ investigated the sound generated in a separated shear flow by flow visualization and fluctuating static pressure measurements, and the results showed that the dilatation theory's source term and the vortical structure are closely correlated. Escobar et al. ${ }^{10}$ presented a study on vortex sound propagation by using finite element method and compared dilatation theory with Lighthill's acoustic analogy theory. The results showed that the dilatation theory can get good solutions easily. Based on the dilatation theory, Papageorgakopoulos and Tsangaris ${ }^{11}$ developed a numerical discretization scheme for acoustic wave equation and solved some benchmark problems.

Computational Aeroacoustics (CAA) is not the same as Computational Fluid Dynamics (CFD). Needs of accurate and efficient numerical solvers in CAA motivated the development of low-dispersion and low-dissipation schemes. Varieties of finite difference schemes ${ }^{12-14}$ occupy a dominant position in CAA and some of them can provide certain accuracy. However, the spectral element method (SEM) can provide high resolution and good flexibility with a low number of elements.
Numerically, SEM has the advantage of low dispersion and diffusion alongside exponential convergence in the polynomial order. Therefore, the SEM has been widely used in wave propagation studies, ${ }^{15,16} \mathrm{CFD}^{17}$ and CAA. ${ }^{18-20}$

The motivation for simulating the sound generated by a corotating vortex pair ${ }^{5,21-23}$ in this work is that various vortices occupy only a very small portion in a flow but play a key role in organizing the flow, ${ }^{24}$ as "the sinews and muscles of the fluid motion" 25 and "the sinews of turbulence". ${ }^{26}$ Vortices are also "the voice of fluid motion" 27 because they, at low Mach numbers, are the only source of aeroacoustic sound and noise. The other reason is that it has analytic solutions. Moreover, many other workers have used it to verify the validity of the proposed numerical schemes for CAA. ${ }^{28-31}$

In the present work, we present an analysis methodology, described in Section 2, which aims to supply another computational tool for CAA. The inhomogeneous convected wave equation based on the dilatation theory was solved numerically by using the high resolution SEM. In order to demonstrate the capabilities and limits of this method, we have studied a benchmark 2D vortex sound propagation problem in detail.

The paper is organized as follows. In Section 2 we provide a description of the inhomogeneous convected wave equation with dilatation theory. The governing equation obtained will then be discretized by SEM in space and Newmark- $\beta$ method in time. In Section 3, the sound generated by a co-rotating vortex pair is investigated in detail. Finally, Section 4 contains the conclusions of our work.

\section{GOVERNING EQUATION}

As well known, the original Lighthill's acoustic analogy completely ignores the mean flow-sound interaction effects. Then Phillips and Lilley make a correction for the Lighthill's equation. Phillips' equation takes into account partially the interaction of the mean flow with the sound. Thus, the equa- 
tion is valid for a moving medium, with some accuracy. Lilley's equation takes the effects of the static flow into account in a better way than the Phillips' equation. In Lilley's equation, all the "propagation effects" that occur in a transversely sheared mean flow are inside the left hand side of the equation. Therefore, Lilley's equation has been extensively used to examine the mean flow-sound interaction and for calculation of subsonic jet noise. However, the complexity of solutions and nonlinearity of equations has also been presented. Hence, in the present paper, we consider the Lighthill's acoustic analogy theory in a mean flow velocity $U$ which is constant and parallel to the $x$ direction. For the simplicity of illustration, the Lighthill's equation can be written as

$$
\frac{1}{c_{0}^{2}} \frac{D^{2} p^{\prime}}{D t^{2}}-\frac{\partial^{2} p^{\prime}}{\partial x_{i}^{2}}=\frac{\partial^{2} T_{i j}}{\partial x_{i} x_{j}} ;
$$

where

$$
\frac{D}{D t}=\frac{\partial}{\partial t}+U \frac{\partial}{\partial x}
$$

and $c_{0}, U$ are the speed of sound and mean flow, respectively, $p^{\prime}$ is the pressure fluctuation, $T_{i j}$ represents the Lighthill's stress tensor.

Some assumptions are made here to obtain Eq. (1):

- The speed of sound is a constant.

- There are no mass, heat, force or momentum source distributions.

- The refraction effects are not included in the scope of this paper.

- The sources were assumed to be acoustically compact.

For the inviscid flow with a low Mach number and high Reynolds number in the form

$$
T_{i j} \cong \rho_{0} u_{i} u_{j}
$$

and $u_{i}$ is the fluid velocity of the $i$-direction. Introducing Mach number $M a=U / c_{0}$, Eq. (1) can be written as

$$
\begin{aligned}
\frac{1}{c_{0}^{2}} \frac{\partial^{2} p^{\prime}}{\partial t^{2}}+ & 2 \frac{M a}{c_{0}} \frac{\partial}{\partial x}\left(\frac{\partial p^{\prime}}{\partial t}\right)- \\
& \left(\left(1-M a^{2}\right) \frac{\partial^{2} p^{\prime}}{\partial x^{2}}+\frac{\partial^{2} p^{\prime}}{\partial y^{2}}\right)=\frac{\partial^{2} T_{i j}}{\partial x_{i} x_{j}} .
\end{aligned}
$$

The pressure fluctuations will therefore satisfy the above inhomogeneous convected wave equation which is an exact consequence of the continuity and momentum equations. However, if the flow is incompressible, the pressure fluctuations can be split into the pseudo-sound and acoustic part

$$
p^{\prime}=p^{i n c}+p^{a} .
$$

The pseudo-sound pressure is also called hydrodynamic pressure fluctuations or incompressible pressure fluctuations. The acoustic perturbations are what we term sound, as they are characterized by their ability to propagate (not the generation) into the hearing region. The pseudo-sound pressure perturbations are the consequence of fluid flow simply changing in the source region, it exhibits no wave propagation. The $p^{i n c}$ field dominating within and near the turbulence at subsonic speeds,

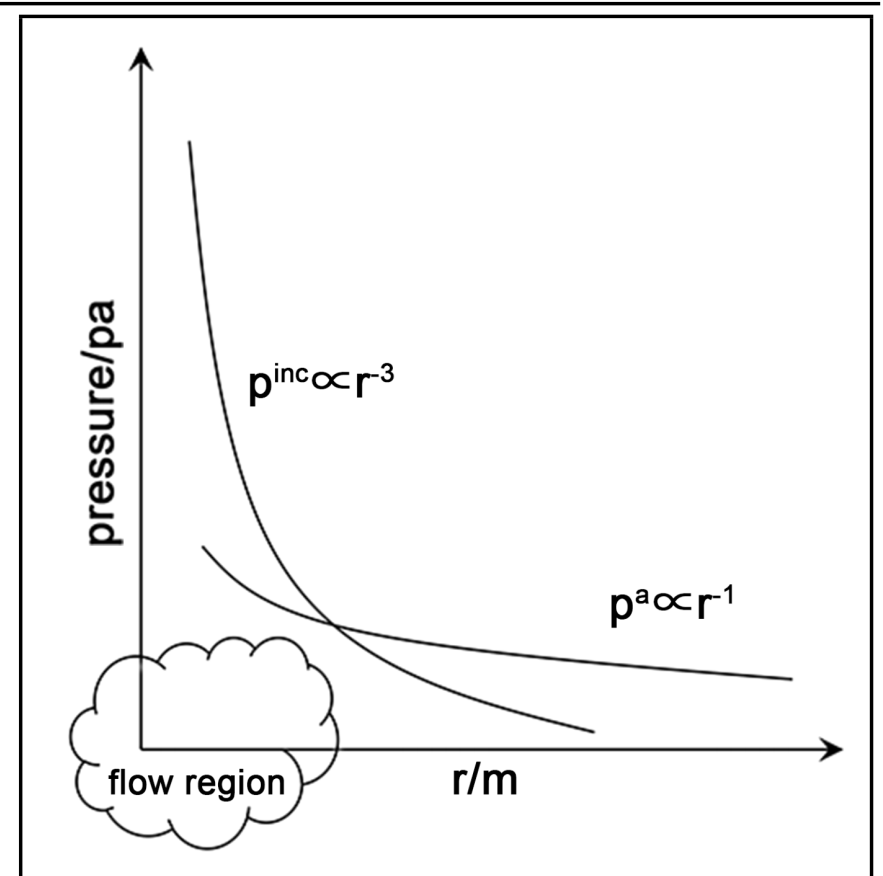

Figure 1. Diagram of decay for acoustic and pseudo-sound pressure in 3-D problem.

constitutes what is known as the acoustic near field, and it has virtually the characteristics of the pressure field in an incompressible flow being dominated by inertial rather than compressional effects, and hence the name pseudo-sound. ${ }^{3}$ Further, it is overridden by the acoustic radiation field $p^{a}$, which decays more slowly with distance. Figure 1 shows the diagram of decay for acoustic and pseudo-sound pressure in 3-D problem. And it has also been suggested by Ribner, ${ }^{2} \mathrm{Crawley}^{7}$ and Ristorcelli. ${ }^{32}$ The horizontal axis represents the distance from the sound source, $r / m$.

Finally, by taking the divergence of momentum equation and combined with the time derivative of the continuity equation, we can get a specific equation for the pseudo-sound pressure $p^{i n c}$

$$
-\nabla^{2} p^{i n c}=\rho_{0} \frac{\partial^{2} u_{i} u_{j}}{\partial x_{i} x_{j}}
$$

and substitute back in Eq. (1) and use Eq. (5) to get

$$
\frac{1}{c_{0}^{2}} \frac{D^{2} p^{a}}{D t^{2}}-\frac{\partial^{2} p^{a}}{\partial x_{i}^{2}}=-\frac{1}{c_{0}^{2}} \frac{D^{2} p^{i n c}}{D t^{2}} .
$$

The above equation can be rewritten as

$$
\begin{aligned}
\frac{\partial^{2} p^{a}}{\partial t^{2}}+ & 2 c_{0} M a \frac{\partial}{\partial x}\left(\frac{\partial p^{a}}{\partial t}\right)- \\
& c_{0}^{2}\left(\left(1-M a^{2}\right) \frac{\partial^{2} p^{a}}{\partial x^{2}}+\frac{\partial^{2} p^{a}}{\partial y^{2}}\right)=-\frac{\partial^{2} p^{i n c}}{\partial t^{2}} .
\end{aligned}
$$

Equation (8) provides another view of getting acoustic pressure by using only the second derivative of pseudo-sound pressure. This formulation corresponds to what derived by Hardin and Pope. ${ }^{5}$ The right hand side of Eq. (8) can be regarded as the forcing term of the inhomogeneous convected wave equation for the acoustic pressure. And like Lighthill's result, the above equation is an exact consequence of the continuity and momentum equations. The initial condition is given by

$$
p^{a}(x, y, 0)=0, \quad \frac{\partial p^{a}}{\partial t}(x, y, 0)=0 .
$$




\subsection{Nonreflecting Boundary Condition}

One of the typical problems in the numerical simulation of acoustic wave propagation is the treatment of boundary conditions. The nonreflecting boundary conditions are introduced to suppress the spurious waves which are generated by truncating the unbounded domain, and this would lead to a significant reduction in computational cost, especially for the threedimensional large-scale numerical simulation.

In this study, the C-E-M nonreflecting boundary condition is modified to be appropriate for the convective wave equation. ${ }^{33-35}$ The expression appears as what shown below

$$
\frac{1}{c_{0}(1 \mp M a)} \frac{\partial p^{a}}{\partial t}+\frac{\partial p^{a}}{\partial \mathbf{n}}=0
$$

where $\mathbf{n}$ represents the outward unit normal vector out of the computation region at the boundaries. With a minus sign placed in front of $M a$ on the upstream boundary and a plus sign on the downstream boundary, the $M a$ is zero on the boundary perpendicular to the flow direction.

\subsection{Spatial Discretization}

Both integral formula and variational formula can be applied to the governing equation. Because they are computationally less expensive than volume discretization methods where a whole discretization of the acoustic domain is required, the integral methods remain widely used in CAA studies, but in such a situation, integral formula would require extensive experience of a hard-wall Green's function that is not known for complex geometries. On the other hand, the integral formula must explicitly take into account the interactions between the solid surfaces and the induced noise which is named as surface sources. On the contrary, the variational formula is able to take into account the effects implicitly. And therefore, in the present paper, we adopt the SEM to discretize the governing equation in space.

After introducing the Sobolev space ( $d$ denotes the space dimension)

$$
H^{1}(\Omega):=\left\{v \in L^{2}(\Omega): \frac{\partial v}{\partial x_{j}} \in L^{2}(\Omega), \forall j=1, \ldots d\right\}
$$

and its subspace

$$
H_{0}^{1}(\Omega):=\left\{v \in H^{1}(\Omega):\left.v\right|_{\Gamma_{D}=0}\right\} .
$$

Then, the weak formulation of Eq. (8) and Eq. (9) reads as follows: find $p^{a}(t) \in H^{1}(\Omega)$ such that, for any $v \in H_{0}^{1}(\Omega)$,

$$
\begin{aligned}
& \iint_{\Omega}\left(\frac{\partial^{2} p^{a}}{\partial t^{2}}+2 c_{0} M a \frac{\partial}{\partial x}\left(\frac{\partial p^{a}}{\partial t}\right)-\right. \\
& \left.c_{0}^{2}\left(\left(1-M a^{2}\right) \frac{\partial^{2} p^{a}}{\partial x^{2}}+\frac{\partial^{2} p^{a}}{\partial y^{2}}\right)\right) v d \Omega=\iint_{\Omega}-\frac{\partial^{2} p^{i n c}}{\partial t^{2}} v d \Omega .
\end{aligned}
$$

The variational formulation of above equation with the nonre- flecting boundary condition Eq. (10) yields as follows

$$
\begin{gathered}
\iint_{\Omega} \frac{\partial^{2} p^{a}}{\partial t^{2}} v d \Omega+2 c_{0} M a \iint_{\Omega} \frac{\partial}{\partial x}\left(\frac{\partial p^{a}}{\partial t}\right) v d \Omega+ \\
c_{0}^{2} \iint_{\Omega}\left[\left(1-M a^{2}\right) \frac{\partial p^{a}}{\partial x} \frac{\partial v}{\partial x}+\frac{\partial p^{a}}{\partial y} \frac{\partial v}{\partial y}\right] d \Omega- \\
c_{0} \oint_{\Gamma_{\text {xup }}}(1+M a) \frac{\partial p^{a}}{\partial t} v d s+c_{0} \oint_{\Gamma_{y}} \frac{\partial p^{a}}{\partial t} v d s+ \\
c_{0} \oint_{\Gamma_{\text {xdown }}}(1-M a) \frac{\partial p^{a}}{\partial t} v d s=\iint_{\Omega}-\frac{\partial^{2} p^{i n c}}{\partial t^{2}} v d \Omega .
\end{gathered}
$$

where $\Gamma_{x u p}, \Gamma_{x d o w n}$ and $\Gamma_{y}$ is the upstream boundary, downstream boundary and the boundary in the $y$ direction, respectively.

In the Chebyshev spectral element method, the computational domain $\Omega$ is decomposed into $N_{d}=N_{m} \times N_{n}$ nonoverlapping subdomains, where $N_{m}$ the element number in the $x$ direction, $N_{n}$ the element number in the $y$ direction. Each spectral element is mapped into a standard element $[-1,1]$ by

$$
\begin{array}{rlrl}
\xi & =\frac{2}{L_{x}^{i}}\left(x-x_{i}\right)-1 \quad \text { or } & x=\frac{1}{2} L_{x}^{i}(\xi+1)+x_{i} \\
\eta & =\frac{2}{L_{y}^{i}}\left(y-y_{i}\right)-1 \quad \text { or } \quad y=\frac{1}{2} L_{y}^{i}(\eta+1)+y_{i}
\end{array}
$$

where $L_{x}^{i}=x_{m+1}-x_{m}, L_{y}^{i}=y_{n+1}-y_{m}$ are the lengths of the $i$ th element in $x$ and $y$ directions, respectively. Hence, the trial functions and the test functions can be written as

$$
\begin{aligned}
p^{a i}(\xi, \eta) & =\sum_{j=0}^{N_{x}^{i}} \sum_{k=0}^{N_{y}^{i}} h_{j}^{i}(\xi) h_{k}^{i}(\eta) u_{j k}^{i} \\
v^{i}(\xi, \eta) & =\sum_{p=0}^{N_{x}^{i}} \sum_{q=0}^{N_{y}^{i}} h_{p}^{i}(\xi) h_{q}^{i}(\eta) v_{p q}^{i}
\end{aligned}
$$

where $N_{x}^{i}, N_{y}^{i}$ is the number of nodes in each element in $x$ and $y$ directions, respectively. Interpolation functions can be expressed as

$$
\begin{aligned}
& h_{j}^{i}(\xi)=\frac{2}{N_{x}^{i}} \sum_{m=0}^{N_{x}^{i}} \frac{1}{c_{j} c_{m}} T_{m}\left(\xi_{j}^{i}\right) T_{m}(\xi) ; \\
& h_{k}^{i}(\eta)=\frac{2}{N_{y}^{i}} \sum_{n=0}^{N_{y}^{i}} \frac{1}{c_{k} c_{n}} T_{n}\left(\eta_{k}^{i}\right) T_{n}(\eta) ; \\
& h_{p}^{i}(\xi)=\frac{2}{N_{x}^{i}} \sum_{l=0}^{N_{x}^{i}} \frac{1}{c_{p} c_{l}} T_{l}\left(\xi_{p}^{i}\right) T_{l}(\xi) ; \\
& h_{q}^{i}(\eta)=\frac{2}{N_{y}^{i}} \sum_{r=0}^{N_{y}^{i}} \frac{1}{c_{q} c_{r}} T_{r}\left(\eta_{q}^{i}\right) T_{r}(\eta) ;
\end{aligned}
$$

where $T_{m}, T_{n}, T_{l}, T_{r}$ are Chebyshev polynomials. The interpolation functions satisfy the cardinal interpolation property

$$
h_{j}^{i}\left(\xi_{k}\right)=\delta_{j k}, \quad h_{p}^{i}\left(\eta_{q}\right)=\delta_{p q} ;
$$

where $\delta_{j k}, \delta_{p q}$ are Kronecker's deltas representing the identity matrix. The parameter $c_{m}$ is defined by

$$
c_{m}= \begin{cases}2 & m=0, N_{x}^{i} \\ 1 & m \neq 0, N_{x}^{i}\end{cases}
$$


Finally, the variational problem discretized by the means of SEM is equivalent to solving the following Differential Algebraic Equations

$$
\mathbf{M} \ddot{p}^{a}(t)+\mathbf{C} \dot{p}^{a}(t)+\mathbf{K} p^{a}(t)=\mathbf{S} ;
$$

where $\mathbf{M}, \mathbf{C}, \mathbf{K}$ and $\mathbf{S}$ are the global mass matrix, global damping matrix, global stiffness matrix and global loading matrix.

\subsection{Time Integration Scheme}

Newmark- $\beta$ scheme is used to avoid the stability restriction. It is an implicit method of direct integration of the equations, for which the relationships between the acoustic pressure $p^{a}$, the first time derivative $\dot{p}^{a}$ and the second time derivative $\ddot{p}^{a}$ in the interval of $t \sim t+\Delta t$ are as follows:

$$
\begin{aligned}
& \dot{p}_{t+\Delta t}^{a}=\dot{p}_{t}^{a}+(1-\gamma) \ddot{p}_{t}^{a} \Delta t+\gamma \ddot{p}_{t+\Delta t}^{a} \Delta t \\
& p_{t+\Delta t}^{a}=p_{t}^{a}+\dot{p}_{t}^{a} \Delta t+\left(\frac{1}{2}-\beta\right) \ddot{p}_{t}^{a} \Delta t^{2}+\beta \ddot{p}_{t+\Delta t}^{a} \Delta t^{2}
\end{aligned}
$$

The choice of $\gamma$ and $\beta$ in the equations above will influence the stability and accuracy of the method. ${ }^{36}$ After utilising the above relationships, Eq. (25) reduces to a system of algebraic equations with constant coefficients for each given time step $\Delta t$, the obtained form can be written as

$$
\begin{aligned}
& \left(\mathbf{K}+\frac{1}{\beta \Delta t^{2}} \mathbf{M}+\frac{\gamma}{\beta \Delta t} \mathbf{C}\right) p_{t+\Delta t}^{a}= \\
& \mathbf{S}_{t+\Delta t}+\mathbf{M}\left[\frac{1}{\beta \Delta t^{2}} p_{t}^{a}+\frac{1}{\beta \Delta t} \dot{p}_{t}^{a}+\left(\frac{1}{2 \beta}-1\right) \ddot{p}_{t}^{a}\right]+ \\
& \mathbf{C}\left[\frac{\gamma}{\beta \Delta t} p_{t}^{a}+\left(\frac{\gamma}{\beta}-1\right) \dot{p}_{t}^{a}+\left(\frac{\gamma}{2 \beta}-1\right) \Delta t \ddot{p}_{t}^{a}\right]
\end{aligned}
$$

and the initial step of is written as follows:

$$
\ddot{p}_{0}^{a}=\mathbf{M}^{-1}\left(\mathbf{S}_{0}-\mathbf{K} p_{0}^{a}-\mathbf{C} \dot{p}_{0}^{a}\right) .
$$

\section{RESULTS AND DISCUSSION}

In this paper, the acoustic field generated by a co-rotating vortex pair is calculated in detail, and can be used as a benchmark problem to verify computational aeroacoustics numerical schemes. This vortex sound problem is a good test for the algorithm because an analytical closed form solution exists for both the incompressible flow field and the acoustic field. At the same time, it also represents the basic acoustic field generated by turbulent shear flows and can be used to understand the mechanism of sound generation.

The schematic of the co-rotating vortices is presented in Fig. 2. The two point-vortices separated by a fixed distance $2 r_{0}$ rotate around each other along a circular path of radius $r_{0}$ and have an equal circulation intensity $\Gamma$. The angular rotational speed is $\omega=\Gamma /\left(4 \pi r_{0}^{2}\right)$, and the period of rotation is $T=8 \pi^{2} r_{0}^{2} / \Gamma$. Each vortex induces on the other a velocity $v_{\theta}=\Gamma /\left(4 \pi r_{0}\right)$, thus the rotating Mach number is $M_{r}=v_{\theta} / c_{0}$.

The flow field is assumed to be viscous and incompressible. Thus, it can be determined numerically by the evaluation of a complex potential function $\phi(z, t)$

$$
\phi(z, t)=\frac{\Gamma}{2 \pi i} \ln z^{2}\left(1-\frac{b^{2}}{z^{2}}\right) ;
$$

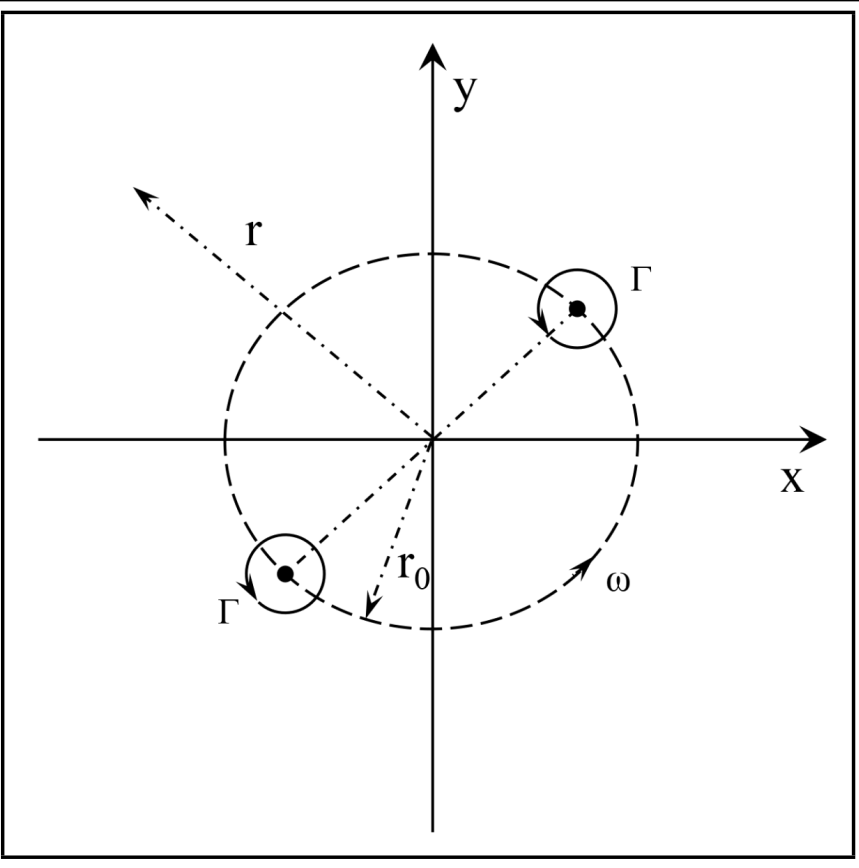

Figure 2. The schematic of the co-rotating vortex pair.

where $z=x+i y=r e^{i \theta}, b=r_{0} e^{i \omega t}$.

To make acoustic computations, flow variables are required as input to the acoustic equations. The hydrodynamic velocity can be obtained by differentiating Eq. (30) with respect to $z$. From the unsteady Bernoulli's equation, the hydrodynamic pressure $p^{i n c}$ can be found as follow

$$
\begin{gathered}
u_{x}-i u_{y}=\frac{\partial \phi(z, t)}{\partial z}=\frac{\Gamma}{i \pi} \frac{z}{z^{2}-b^{2}} \\
p^{i n c}=p_{0}-\rho_{0} \frac{\partial}{\partial t}\{\operatorname{Re}(\phi(z, t))\}-\frac{1}{2} \rho_{0}\left(u_{x}^{2}+u_{y}^{2}\right)
\end{gathered}
$$

where Re denotes the real part of a complex quantity.

The inherent unsteadiness of the flow field of the co-rotating vortex pair generates sound. The analytical solution by Müller and Obermeier ${ }^{27}$ is used to validate the numerical simulation. The fluctuating pressure is given by

$$
p^{a}=\frac{\rho_{0} \Gamma^{4}}{64 \pi^{3} r_{0}^{4} c_{0}^{2}}\left[J_{2}(k r) \sin (\psi)-Y_{2}(k r) \cos (\psi)\right] ;
$$

where wave number $k=2 \omega / c_{0}$ and $J_{2}(k r), Y_{2}(k r)$ are the second-order Bessel function of the first and second kind, respectively.

The acoustic computations are performed in the domain $L * L$ with the uniform square grid system. The flow domain, the same as the acoustic source region, corresponds to the inner square domain with dimensions $0.5 L * 0.5 L$. Zero initial values are used for all acoustic fluctuations. The circulation intensity and the rotating Mach number are the only parameters that determine the frequency and amplitude of the solution. The parameters used for the following simulation are $\Gamma=1.00531 \mathrm{~m}^{2} \mathrm{~s}^{-1}, r_{0}=1 \mathrm{~m}, c_{0}=1 \mathrm{~ms}^{-1}, M a=0$, $L=400 \mathrm{~m}, N_{m}=N_{n}=55, N_{x}=N_{y}=2$, this results in a wave length $\lambda \approx 39 \mathrm{~m}, M_{r}=0.08$ and the element size $\Delta x=\Delta y=3.64 \mathrm{~m}$, corresponding to about 11 points per wavelength (PPW), time step $\Delta t=0.1 \mathrm{~s}$.

Figure 3 shows a 3-D graphical view of the acoustic pressure analytical solution. As what can be seen from the graph 


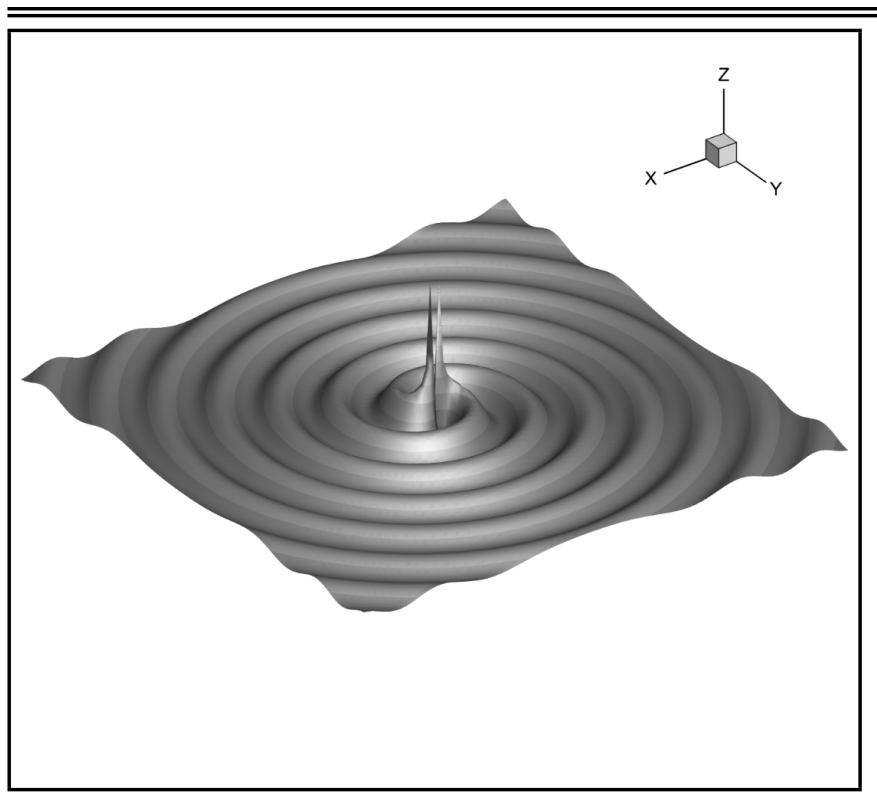

Figure 3. 3-D graphical view of the acoustic pressure analytical solution.

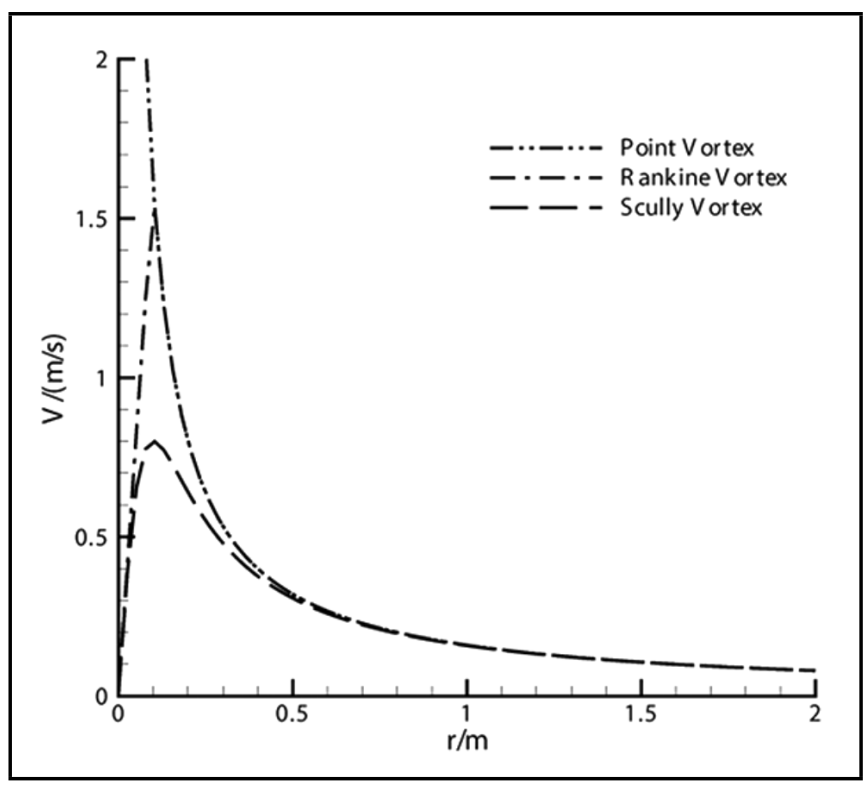

Figure 4. Vortex tangential velocity variation against the radial distance.

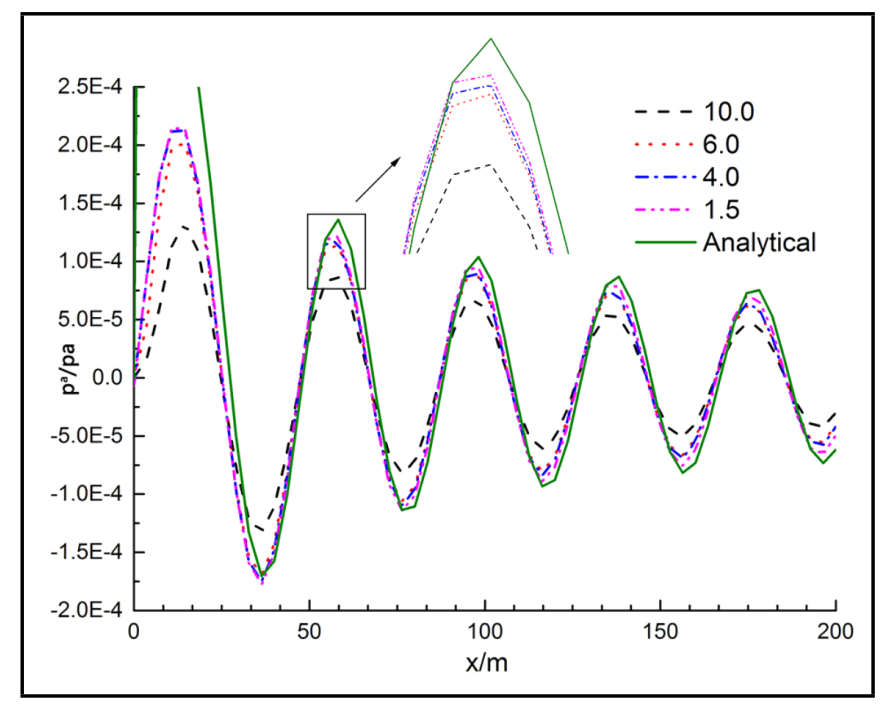

Figure 5. Comparison of the numerical and analytical acoustic pressure solutions along the positive horizontal $x$-axis with different cut-off distances.

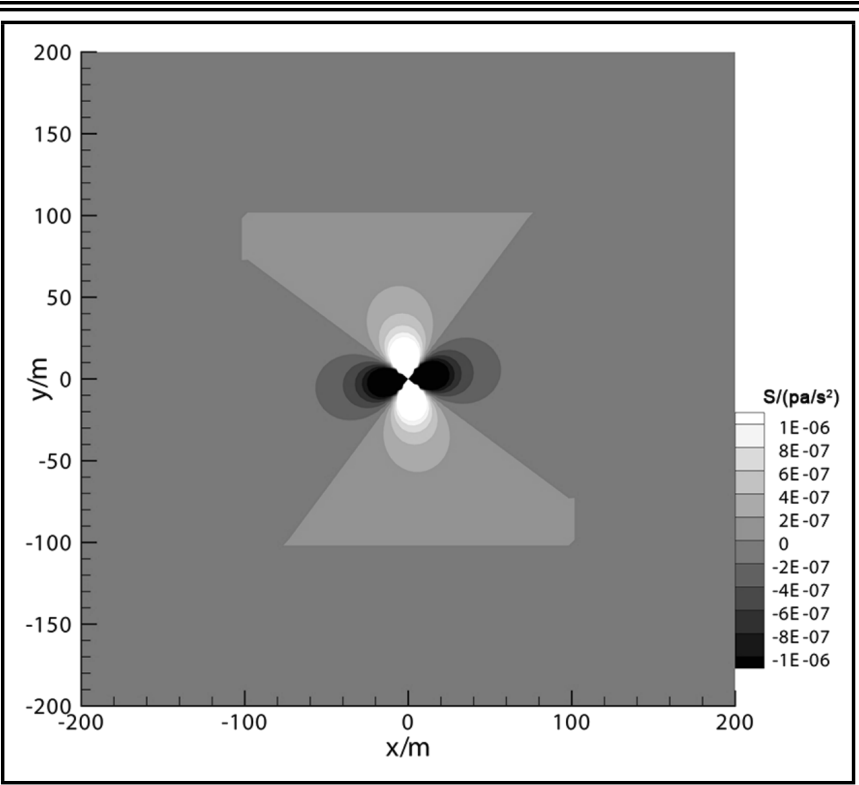

Figure 6. Source term distribution at time $100 \mathrm{~s}$.

above, the double spiral pattern clearly illustrates the rotating quadrupole nature of the radiated waves, and the acoustic pressure becomes singular at the coordinate origin and has large gradients close to the vortex centers. To avoid the numerical singularity at the center of the vortices, the vortex core model ${ }^{20}$ is used, and the other method involves placing the mesh points far enough from the vortex centers. ${ }^{22}$ The original point vortex, Rankine vortex and Scully vortex tangential velocity variation against the radial distance are plotted in Fig. 4. A cut-off practice was adopted in this study. The effect of considering different cut-off distances at the coordinate origin is illustrated in Fig. 5. The four different distances from the origin are: $r / r_{0}=1.5,4.0,6.0$ and 10.0, respectively. Figure 5 shows that the amplitude of acoustic pressure is directly associated with the cut-off distance. And with the decreasing of cut-off distances, the numerical solutions are getting better and better. The main reason for this phenomenon is that the bigger cut-off distance has reduced the source terms more. However, for grid points located closer than the point vortex separation distance $r_{0}$, convergence will not obtain on account of large velocity gradients. In addition, on account of the fact that the element size we select is not fine enough, thus for grid points located at distances $r / r_{0} \leq 1.5$, no source term (the right-hand side of Eq. (8)) was computed. The source term distribution acquired from the computation at time $t=100 \mathrm{~s}$ is presented in Fig. 6. As can be seen from the Fig. 6, the source region is large enough to avoid obvious truncation of the source term. The amplitudes on the boundary are about $2.5 \%$ of their peak in the source domain.

A grid resolution study is investigated. Figure 7 a shows the comparison of the numerical and analytical acoustic pressure solutions along the positive horizontal $x$-axis obtained with three different grid spacings. Good agreement is observed except near the center of the vortices due to the source term's cut-off. It is shown that using a grid spacing of $\Delta x=3.92 \mathrm{~m}$, with about $10 \mathrm{PPW}$, provides as good a result as those obtained for the case of $\Delta x=2.82 \mathrm{~m}$, with about 14 PPW, except near the center of the vortices. Hence grid independency is achieved with $\Delta x=3.92 \mathrm{~m}$. Additionally, the effect of temporal resolution is given in Fig. 7b. It is easy to see that the accuracy 


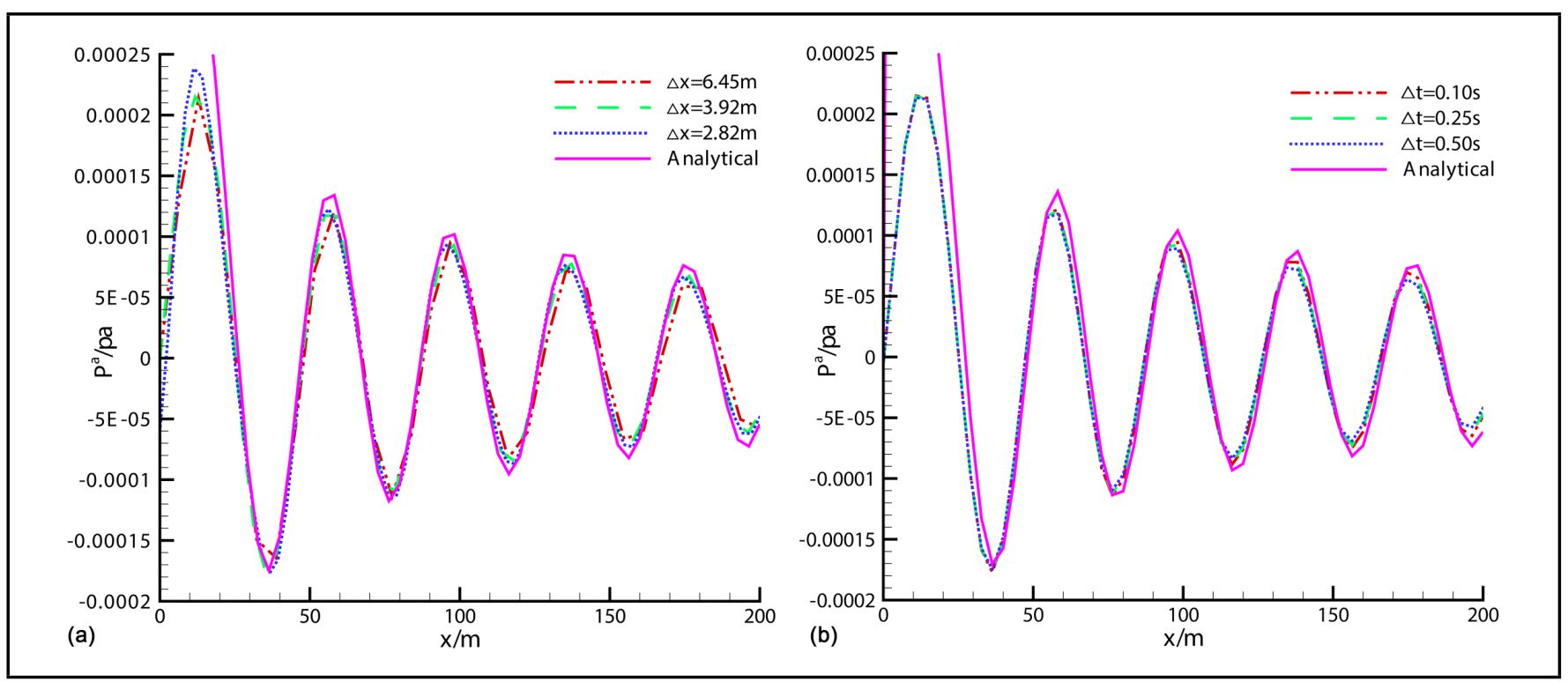

Figure 7. Comparison of the numerical and analytical acoustic pressure solutions along the positive horizontal x-axis (a) different grid spacing and (b) different time step.

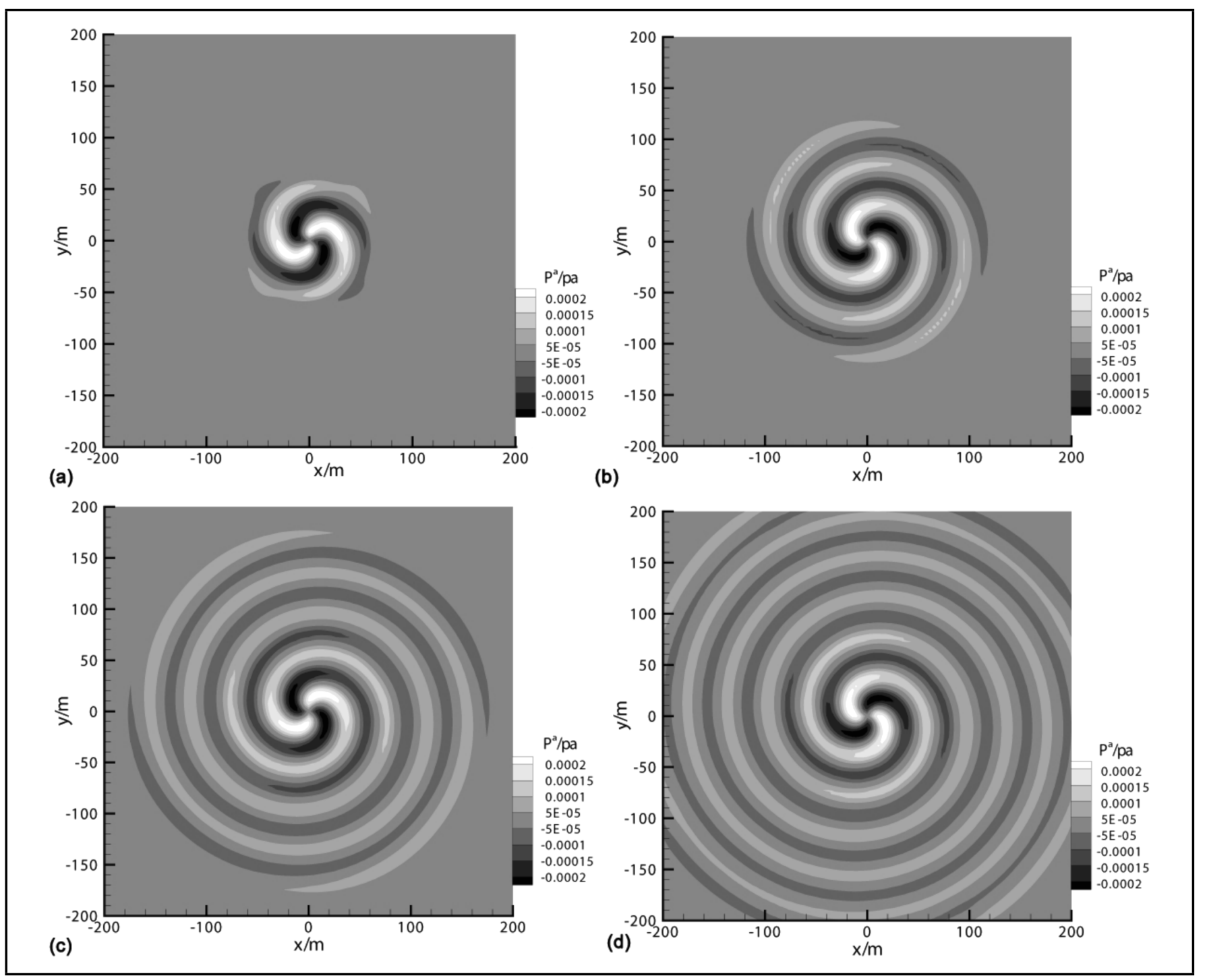

Figure 8. Time evolution of predicted acoustic pressure contours in the stationary medium (a) $60 \mathrm{~s}$, (b) $120 \mathrm{~s}$, (c) $180 \mathrm{~s}$, and (d) $240 \mathrm{~s}$. 


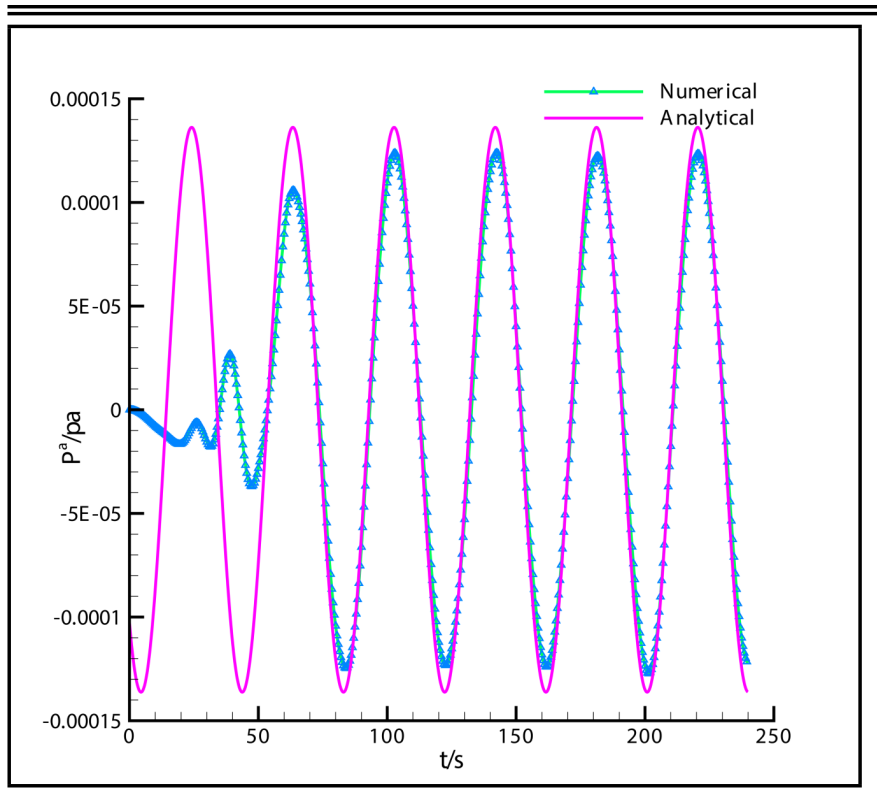

Figure 9. Pressure time series at the monitor point $(0,58.2)$.

enhanced with the shortening of the time step, which means the smaller time step, the better prediction of the amplitudes and phase.

The time evolution of predicted acoustic pressure distributions in the stationary medium is presented in Fig. 8. It can be indicated that the nonreflecting boundary condition worked well for wave propagation in unbounded domains.

The pressure signal is recorded at the monitor point $(0,58.2)$ in order to analyze the radiating frequency, its evolution is reported in Fig. 9. It can be indicated that there is only some slight difference between the amplitudes of the numerical solutions and the analytical one, which could be owing to the neglect of the source terms near the vortex centers.

The effect of considering different rotating Mach number is illustrated in Fig. 10. The parameters used for this simulation are $L=200 \mathrm{~m}, \Delta x=\Delta y=3.33 \mathrm{~m}, \Delta t=0.1 \mathrm{~s}, M_{r}=0.03$, 0.08 and 0.13 , respectively. It shows that the intensity of the sound source is directly associated with the $M_{r}$. Moreover, a higher Mach number means shorter wavelengths with a lower spatial resolution of the waves when the space discretization is not changed. In this case, a finer mesh discretization is needed to resolve this problem.

To evaluate the frequency content of the sound generated by the vortex pair, a fast Fourier transform algorithm was done. Acoustic results of the three different rotating Mach number given above are compared in Fig. 11 in terms of sound pressure levels (SPL) radiated at the monitor point $(0,58.2)$. The agreement is excellent at the fundamental frequency. But in other frequencies, the amplitudes of the numerical solutions are mostly lower than the analytical solutions, which could also be due to the neglect of the source terms near the vortex centers. In addition, the fundamental frequency is increased as the $M_{r}$ increases. Higher harmonics are seen in numerical solutions, but they don't appear in analytical ones.

There are numerous situations, however, where the surrounding medium is more nearly in a state of motion. Therefore, we now consider the situations with a uniform flow and a sheared mean flow and discuss the behavior of the solution for each case. All the other parameters remain the same while the Mach number is changed into $M_{r}=0.1$ and 0.3 in uni-

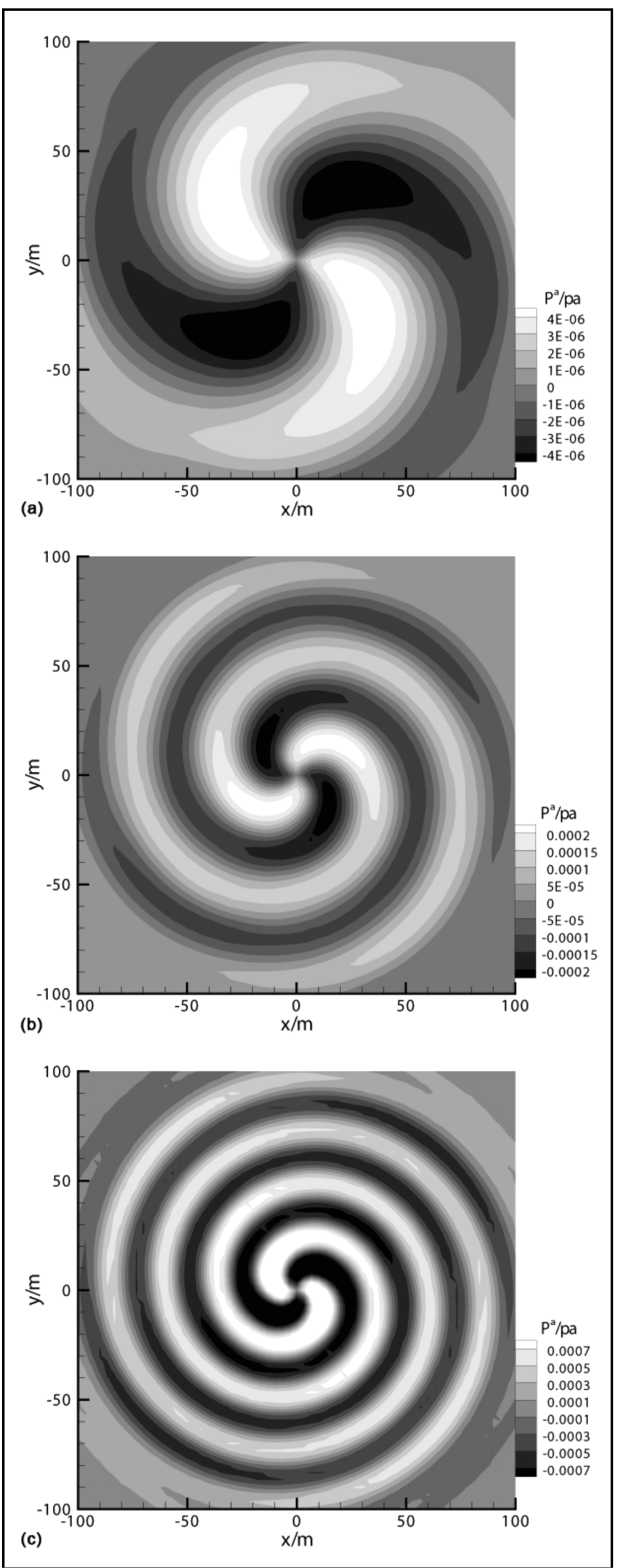

Figure 10. Effect of considering different rotating Mach number (a) $M_{r}=$ 0.03 , (b) $M_{r}=0.08$, and (c) $M_{r}=0.13$. 


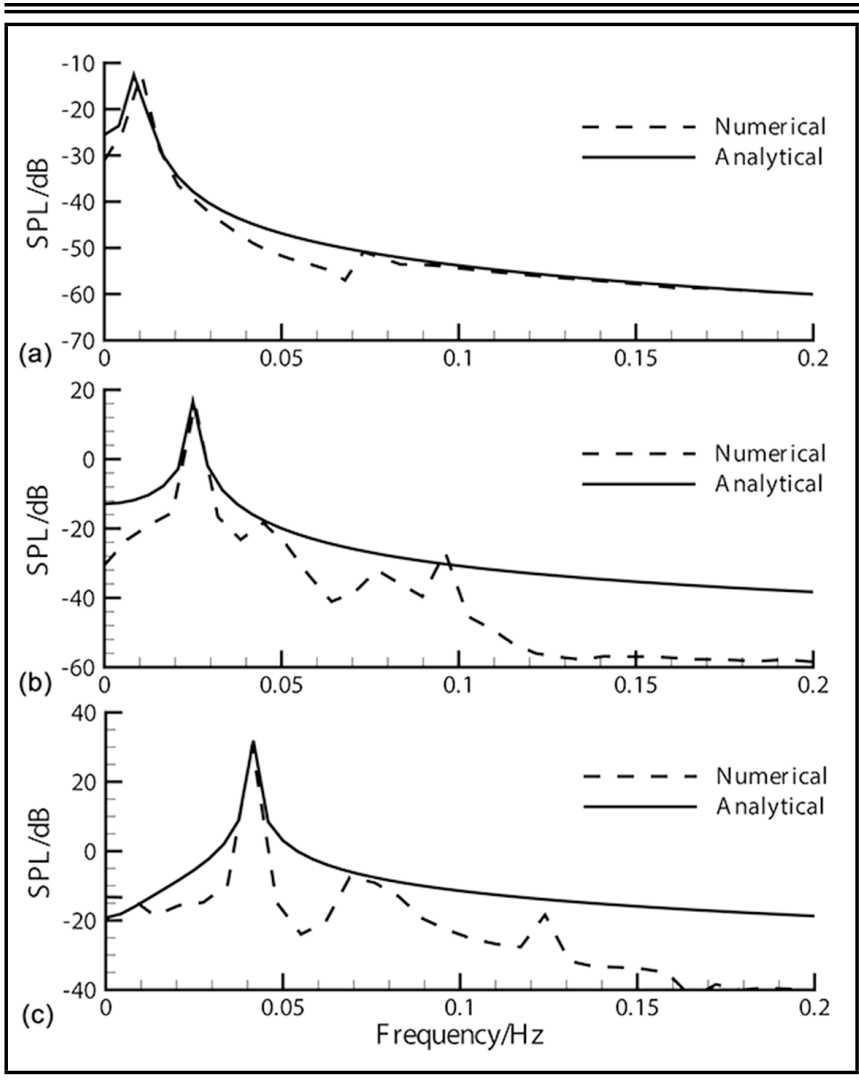

Figure 11. Sound pressure level radiated at point $(0,58.2)$ with three different rotating Mach number (a) $M_{r}=0.03$, (b) $M_{r}=0.08$, and (c) $M_{r}=0.13$.

form mean flow situations. The acoustic pressure contours generated by the vortex pair in the uniform mean flow at time $100 \mathrm{~s}$ is shown in Fig. 12. It can be indicated that the Doppler effects are well captured, which means that the amplitude of the waves increases and their wavelength decreases in the upstream regions. Meanwhile, it is opposite in the downstream regions. Additionally, with the increase of the Mach number, the Doppler effects become increasingly obvious.

Finally, the effects of non-uniform mean flows on acoustic wave propagation are investigated. The shear profile, shown in Fig. 13, is defined by the following hyperbolic tangent expression of the longitudinal mean velocity

$$
u(y)=\Delta U \tanh (2 y / \delta)
$$

where $\Delta U$ and $\delta$ are, respectively, the peak velocity and the shear layer thickness. In the present test, they are chosen to be $\Delta U=0.1 c_{0}$ and $0.3 c_{0}, \delta=10 \mathrm{~m}$. The same computations as in the previous case are performed. Figure 14 shows the acoustic pressure contours generated by the vortex pair in the sheared mean flow at time $100 \mathrm{~s}$. In comparison with Fig. 12, wave fronts are ovalized due to mean flow convection effects.

\section{CONCLUSION}

In the present study, the capabilities of the spectral element method for the accurate simulation of the acoustic field generated by a co-rotating vortex pair using the inhomogeneous convected wave equation combined with Ribner's dilatation theory are investigated. It was observed that the application of the second time derivative of the pseudo-pressure as the source of the inhomogeneous convected wave equation can better simulate the acoustic field propagation. The simulation of consider-

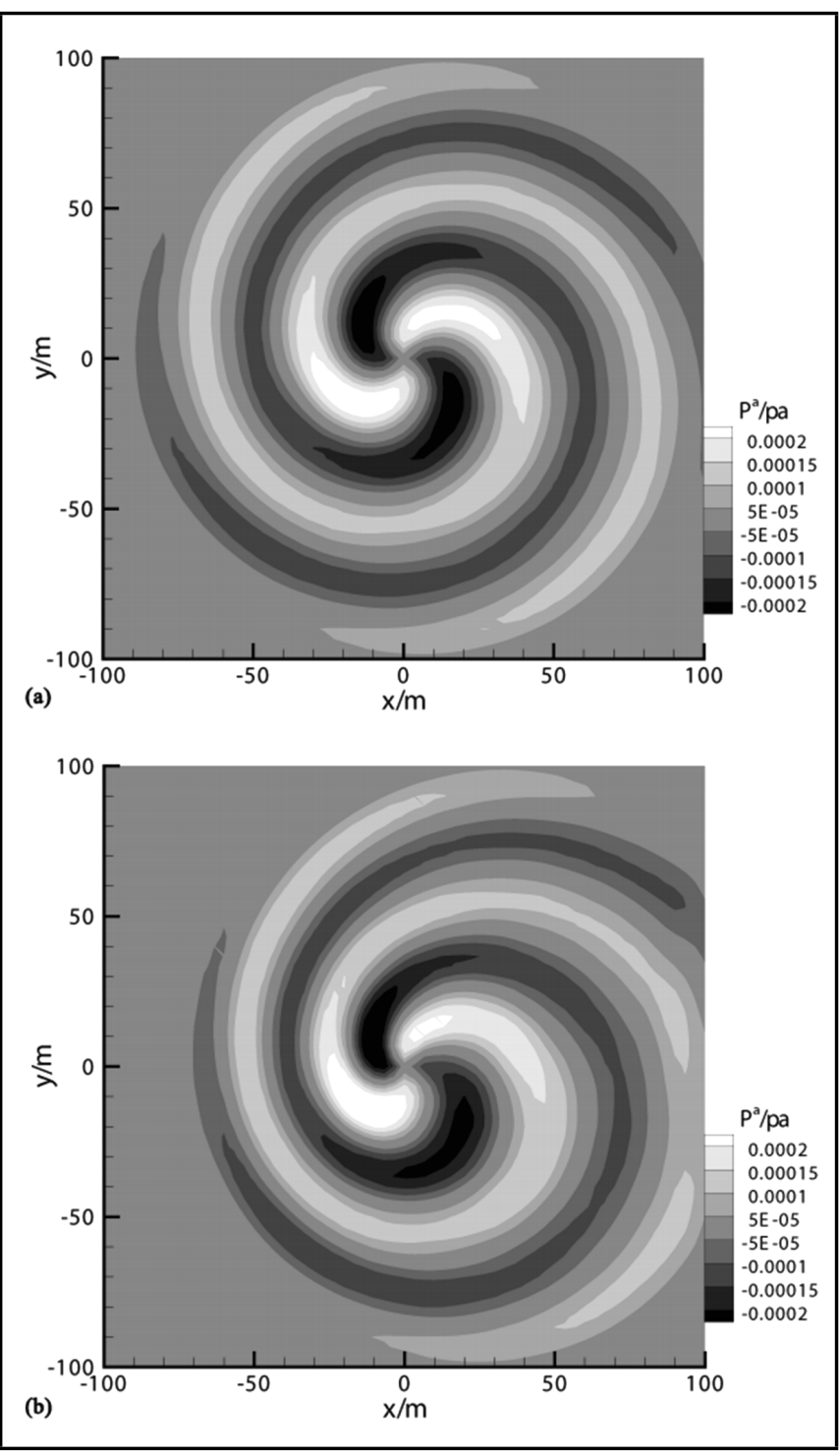

Figure 12. Acoustic pressure contours in the uniform mean flow at time 100s with two different Mach number (a) $M_{r}=0.1$ and (b) $M_{r}=0.3$.

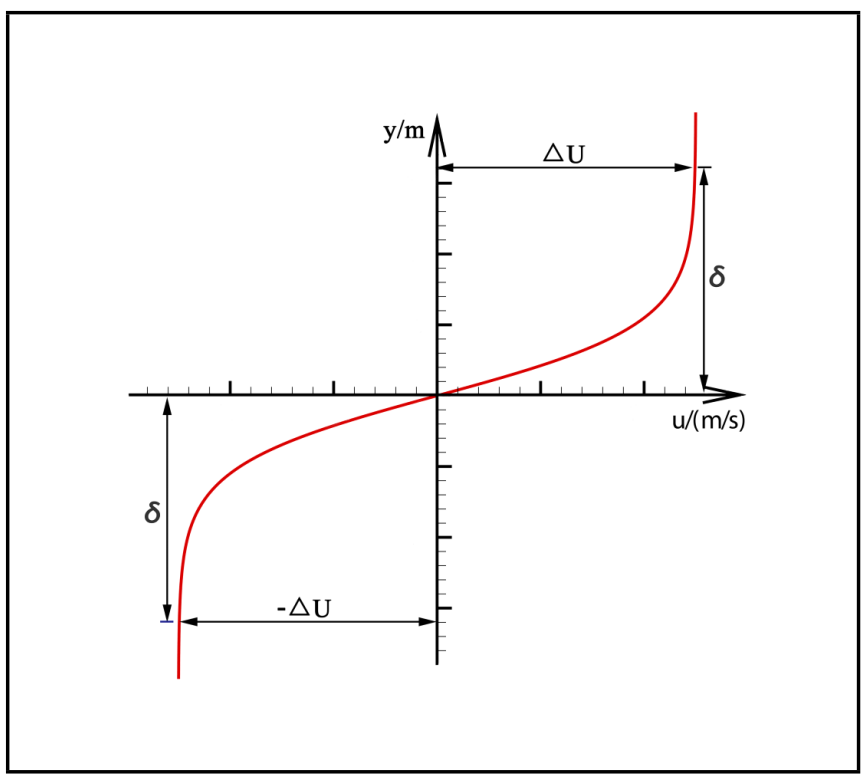

Figure 13. Scheme of the shear layer used for co-rotating vortex pair. 


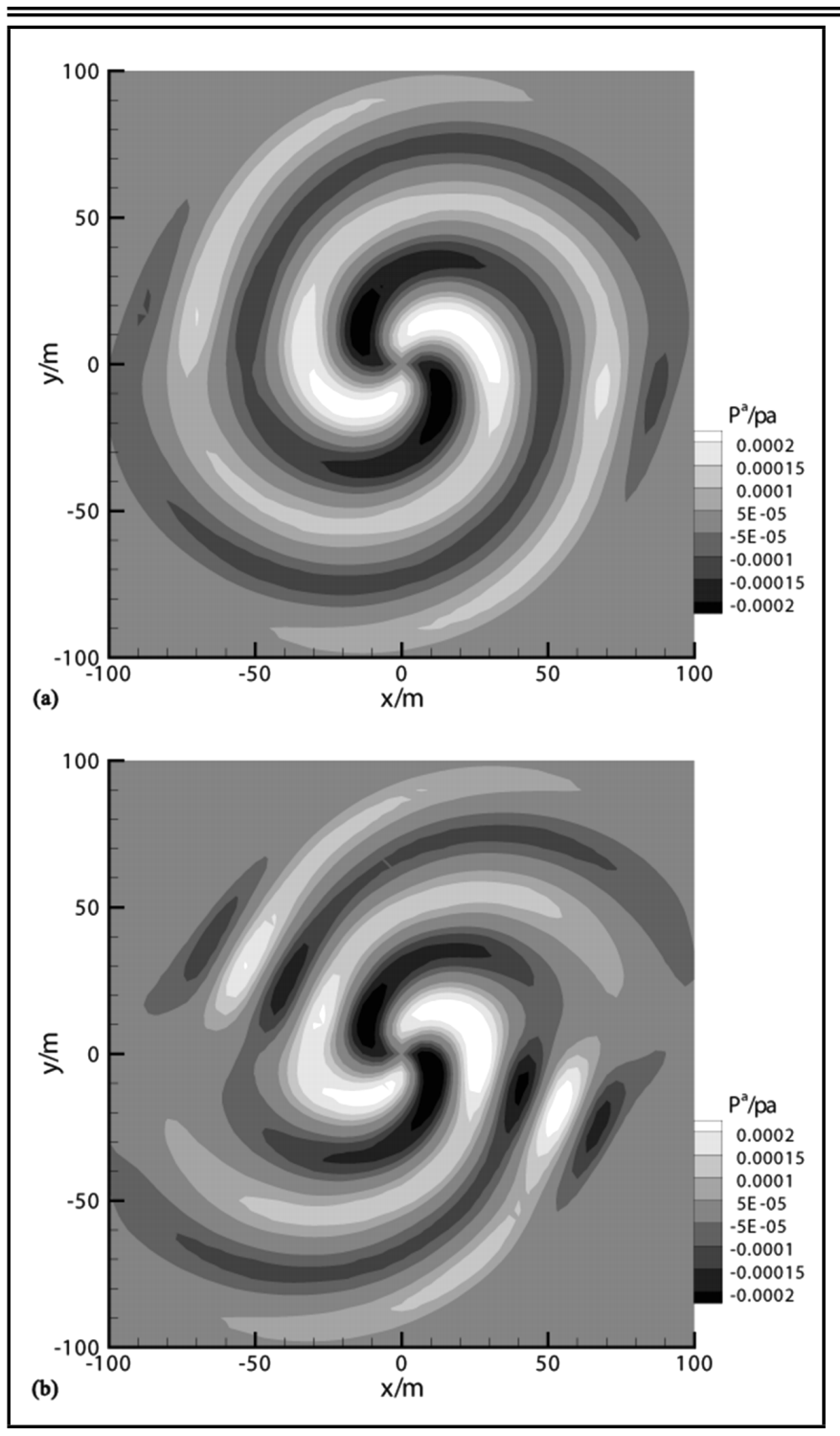

Figure 14. Acoustic pressure contours in the sheared mean flow at time $100 \mathrm{~s}$ with two different Mach number (a) $M_{r}=0.1$ and (b) $M_{r}=0.3$.

ing different grid spacings, time steps and rotating Mach number showed that this method can provide a good solution even on relatively coarse grids and larger time steps. The acoustic pressure amplitude decreased slightly due to the cut off of the sources near the vortex centers, in this case the employment of a vortex core model could provide better results. Additionally, spectral analysis was also considered for evaluating the frequency content of the sound generated by the vortex pair. Moreover, sound propagation in uniform flows and sheared mean flows were simulated and analyzed. Future work will investigate the flow-induced noise problems with the spectral element method and dilatation theory.

\section{ACKNOWLEDGEMENT}

This work was supported by the National Basic Research Program of China [No. 2012CB026004].

\section{REFERENCES}

1 Lighthill, M. J. On sound generated aerodynamically I. General theory, Proceedings of the Royal Society A, 211, 564-587,

(1951) https://dx.doi.org/10.1098/rspa.1952.0060

2 Ribner, H. S. UTIA Rep. 86, AFOSR TN 3430, Aerodynamic sound from fluid dilatations: a theory of the sound from jets and other flows, (1962).

3 Ribner, H. S. The generation of sound by turbulent jets, Advances in Applied Mechanics, 8, 103-182, (1964). https://dx.doi.org/10.1016/s0065-2156(08)70354-5

4 Meecham, W. Discussion of the pressure-source aerosonic theory and of Doak's criticism, The Journal of the Acoustical Society of America, 69 (3), 643-646, (1981). https://dx.doi.org/10.1121/1.385581

5 Hardin, J. C. and Pope, D. S. An acoustic/viscous splitting technique for computational aeroacoustics, Theoretical and Computational Fluid Dynamics, 6, 323-340, (1994). https://dx.doi.org/10.1007/bf00311844

${ }^{6}$ Hurdle, P. M., Meecham W., and Hodder, B. Investigation of the aerodynamic noise generating region of a general electric T-58 jet engine by means of the simple source/fluid dilatation model, The Journal of the Acoustical Society of America, 53, 305-305, (1973). https://dx.doi.org/10.1121/1.1982233

7 Crawley, M. Understanding the aeroacoustic radiation sources and mechanisms in high-speed jets, $\mathrm{PhD}$. thesis, University of Ohio State, (2015).

8 Flemming, F., Sadiki, A., and Janicka, J. Investigation of combustion noise using a LES/CAA hybrid approach, Proceedings of the Combustion Institute, 31, 3189-3196, (2007). https://dx.doi.org/10.1016/j.proci.2006.07.060

9 Hiramoto, R., Toyoda, K., and Sato, K. Study on mechanism of aerodynamic sound generation by flow visualization and fluctuating static pressure measurements, Journal of Fluid Science and Technology, 5 (1), 45-55, (2010). https://dx.doi.org/10.1299/jfst.5.45

10 Escobar, M., Ali, I., Kaltenbacher, M., and Becker, S. Investigation of vortex sound propagation using a FE implementation, 12th AIAA/CEAS Aeroacoustics Conference (27 th AIAA Aeroacoustics Conference), Cambridge, Massachusetts, (2006). https://dx.doi.org/10.2514/6.2006-2518

11 Papageorgakopoulos, J. and Tsangaris, S. A Numerical Method for Predicting Acoustical Wave Propagation in Open Spaces, ISRN Mechanical Engineering, 2011, (2011). https://dx.doi.org/10.5402/2011/174031

12 Cunha, G. and Redonnet, S. On the effective accuracy of spectral-like optimized finite-difference schemes for computational aeroacoustics, Journal of Computational Physics, 263, 222-232, (2014). https://dx.doi.org/10.1016/j.jcp.2014.01.024

13 Liu, W., Kim, J.W., Zhang, X., Angland, D., and Caruelle, B. Landing-gear noise prediction using high-order finite difference schemes, Journal of Sound and Vibration, 332 (14), 3517-3534, (2013). https://dx.doi.org/10.1016/j.jsv.2013.01.035 
14 Liu, X., Zhang, S., Zhang, H., and Shu, C. W. A new class of central compact schemes with spectral-like resolution I: Linear schemes, Journal of Computational Physics, 248, 235-256, (2013). https://dx.doi.org/10.1016/j.jcp.2013.04.014

15 Patera, A. T. A spectral element method for fluid dynamics: laminar flow in a channel expansion, Journal of Computational Physics, 54, 468-488, (1984). https://dx.doi.org/10.1016/0021-9991(84)90128-1

16 Gopalakrishnan, S., Chakraborty, A., and Mahapatra, D. R. Spectral Finite Element Method, Springer-Verlag, London, (2008). https://dx.doi.org/10.1007/978-1-84628-356-7

17 Pozrikidis, C. Introduction to Finite and Spectral Element Methods Using Matlab, Second Edition, CRC Press, Boca Raton, (2014).

18 Rasetarinera, P., Kopriva, D. A., and Hussaini, M. Y. Discontinuous spectral element solution of aeroacoustic problems, Third Computational Aeroacoustics (CAA) Workshop on Benchmark Problems, No. NASA CP-209790, (2000). https://dx.doi.org/10.2514/2.1229

19 Seriani, G. and Oliveira, S. P. DFT modal analysis of spectral element methods for acoustic wave propagation, Journal of Computational Acoustics, 16 (04), 531-561, (2008). https://dx.doi.org/10.1142/s0218396x08003774

20 Zhang, R., Qin, G., and Zhu, C. Spectral element method for acoustic propagation problems based on linearized Euler equations, Journal of Computational Acoustics, 17 (04), 383-402, (2009). https://dx.doi.org/10.1142/s0218396x09004014

21 Lee, D. J. and Koo, S. O. Numerical study of sound generation due to a spinning vortex pair, AIAA Journal, 33, 20-26, (1995). https://dx.doi.org/10.2514/3.12327

22 Prax, C. F., Golanski, F., and Nadal, L. Control of the vorticity mode in the linearized Euler equations for hybrid aeroacoustic prediction, Journal of Computational Physics, 227, 6044-6057, (2008). https://dx.doi.org/10.1016/j.jcp.2008.02.022

23 Ekaterinaris, J. A. New formulation of Hardin-Pope equations for aeroacoustics, AIAA Journal, 37 (9), 1033-1039, (1999). https://dx.doi.org/10.2514/2.829

24 Wu, J. Z., Ma, H. Y., and Zhou, M. D. Vorticity and Vortex Dynamics, Springer-Verlag, Berlin Heidelberg, (2007).

25 Küchemann, D. Report on the IUTAM symposium on concentrated vortex motions in fluids, Journal of Fluid Mechanics, 21 (1), 1-20, (1965). https://dx.doi.org/10.1017/s0022112065000010
26 Moffatt, H., Kida, S., and Ohkitani, K. Stretched vortices-the sinews of turbulence; large-Reynolds-number asymptotics, Journal of Fluid Mechanics, 259, 241-264, (1994). https://dx.doi.org/10.1017/s002211209400011x

27 Müller, E. A. and Obermeier, F. Vortex sound, Fluid Dynamics Research, 3 (1), 43-51, (1988). https://dx.doi.org/10.1016/0169-5983(88)90042-1

28 Hüppe, A. and Kaltenbacher, M. Spectral finite elements for computational aeroacoustics using acoustic perturbation equations, Journal of Computational Acoustics, 20 (2), 113, (2012). https://dx.doi.org/10.1142/s0218396x1240005x

29 Ali, I., Escobar, M., Kaltenbacher, M., and Becker, S. Time domain computation of flow induced sound, Computers \& Fluids, 37 (4), 349-359, (2008). https://dx.doi.org/10.1016/j.compfluid.2007.02.011

30 Kaltenbacher, M., Escobar, M. Becker, S., and Ali, I. Numerical simulation of flow-induced noise using LES/SAS and Lighthill's acoustic analogy, International Journal for Numerical Methods in Fluids, 63 (9), 1103-1122, (2010). https://dx.doi.org/10.1002/fld.2123

31 Zhu, W. J., Shen, W. Z., and Sørensen, J. N. High-order numerical simulations of flow-induced noise, International Journal for Numerical Methods in Fluids, 66 (1), 17-37, (2011). https://dx.doi.org/10.1002/fld.2241

32 Ristorcelli, J. R. Technical Report ICASE-97-21, Fluctuating dilatation rate as an acoustic source, Institute for Computer Applications in Science and Engineering, Hampton, Va, (1997).

33 Clayton, R. and Engquist, B. Absorbing boundary conditions for acoustic and elastic wave equations, Bulletin of the Seismological Society of America, 67 (6), 1529-1540, (1977).

34 Engquist, B. and Majda, A. Absorbing boundary conditions for numerical simulation of waves, Mathematics of Computation, 31 (139), 629-651, (1977). https://dx.doi.org/10.1090/s0025-5718-1977-0436612-4

35 Bécache, E., Givoli, D., and Hagstrom, T. Highorder absorbing boundary conditions for anisotropic and convective wave equations, Journal of Computational Physics, 229, 1099-1129, (2010). https://dx.doi.org/10.1016/j.jcp.2009.10.012

36 Xucheng, W. Finite Element Method, Press of Tsinghua University, Beijing, (2003). 